\title{
Non-Parametric Scan Statistics for Disease Outbreak Detection on Twitter
}

\author{
Feng Chen* and Daniel B. Neill \\ Event and Pattern Detection Laboratory, Carnegie Mellon University, Pittsburgh, PA, USA
}

\section{Objective}

We present a new method for disease outbreak detection, the "Non-Parametric Heterogeneous Graph Scan (NPHGS)". NPHGS enables fast and accurate detection of emerging space-time clusters using Twitter and other social media streams where standard parametric model assumptions are incorrect.

\section{Introduction}

Disease outbreak detection based on traditional surveillance datasets, such as disease cases reported from hospitals, is potentially limited in that the collection of clinic information is costly and time consuming. However, social media provides the vast amount of data available in real time on the internet at almost no cost. Our solution, NPHGS, provides a nonparametric statistical approach for outbreak detection that well addresses the key technical challenges in social media streams.

\section{Methods}

Given Twitter and other social media streams, we first model the streams as a heterogeneous graph, in which: 1) each node can be of different types, such as user, tweet, geographic location, keyword, and hashtag; 2) the relationships between nodes can be different types, such as retweet, reply, and follower; and 3) each node type can have different attributes, such as the number of tweets and users for a given geographic location; the number of followers for a given user; and the number of sentiment score for a given tweet. Second, we further model the network as a "sensor" network, in which each node senses its "neighborhood environment" and reports empirical p-values measuring the current anomalousness levels of various neighborhood-related attributes. Third, we efficiently maximize a nonparametric scan statistic over connected sub-graphs to identify the most anomalous network clusters. Each cluster is returned as the indicator of an ongoing or upcoming outbreak event.

\section{Results}

We randomly selected ten percent of all the raw Twitter data from 2013-January to 2013-June in the country Chile, where 17 rare Hantavirus disease outbreaks in more than eight different states have been collected by Chilean Ministry of Health [1] and also reported in local news reports. For each gold standard event, we determine whether our method: a) Have an alert in the same state 1-7 days before the event, named as successful forecasting. Record the number of days of lead time for that event based on the earliest such alert; b) did not have an alert in that state 1-7 days before the event, but did have an alert in that state 0-7 days after the event, named as successful detection. Record the number of days of lag time for that event based on the earliest such alert; c) otherwise, the alert is regarded as false alarm. Note that, sophisticated preprocesses have been conducted to extract high quality related tweets and to accurately estimate their geographic locations.

Promising results are shown in Figures 1 and 2. First, we observe from Figure 1 that the true positive rate for detection and forecasting reaches $65 \%$ at a false positive rate of 1 per 100 days. The true positive rate for forecasting alone is much lower (about 15\% TPR at
$0.01 \mathrm{FP} /$ day), which suggests that the outbreak signal in Twitter is much weaker prior to the event, i.e., early detection might be a more appropriate use of this data than prediction. Figure 2 shows that at a reasonably low false positive rate of $0.07 \mathrm{FP} /$ day, NPHGS has detection lag time of only 1.5 days, which is better than the typical 3 to 4 days lag time using traditional public health surveillance data.

\section{Conclusions}

NPHGS is a new nonparametric statistical approach for heterogeneous social media streams. The case study on Twitter demonstrates promising results using Twitter data.

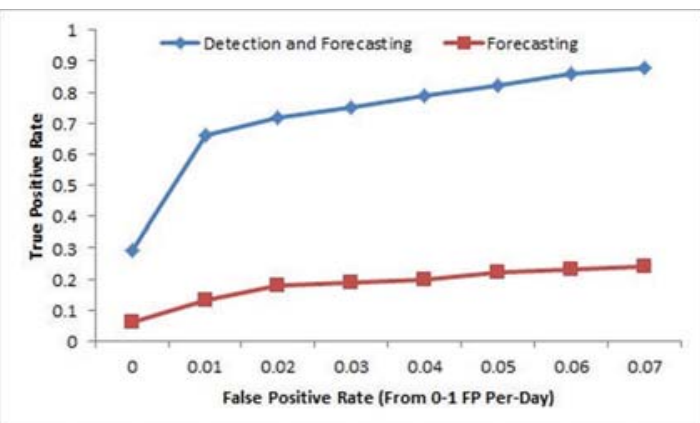

Figure 1: FPR vs. TPR (detection and forecasting)

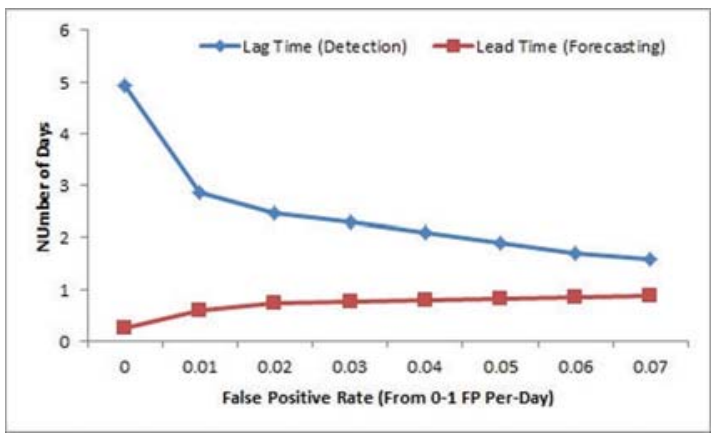

Figure 2: FPR vs. Lead Time and Lag Time

\section{Keywords}

non-parametric scan statistics; social media; disease outbreak

\section{Acknowledgments}

This work was partially supported by the National Science Foundation, grants IIS-0916345, IIS-0911032 and IIS-0953330.

\section{References}

1. http://epi.minsal.cl/vigilancia-epidemiologica/enfermedades-de-notificacion-obligatoria/vigilancia-hantavirus/

\footnotetext{
*Feng Chen

E-mail: fchen1@cmu.edu
} 